\title{
Theory of Frequency-Modulated Combs in Lasers with Spatial Hole Burning, Dispersion, and Kerr Nonlinearity
}

\author{
Nikola Opačak® and Benedikt Schwarz®* \\ Institute of Solid State Electronics, TU Wien, Gusshausstrasse 25-25a, 1040 Vienna, Austria
}

(Received 11 June 2019; revised manuscript received 18 September 2019; published 11 December 2019)

\begin{abstract}
Frequency-modulated (FM) frequency combs constitute an exciting alternative to generate equidistant spectra. The full set of Maxwell-Bloch equations is reduced to a single master equation for lasers with fast gain dynamics to provide insight into the governing mechanisms behind phase locking. It reveals that the recently observed linear frequency chirp is caused by the combined effects of spatial hole burning, group velocity dispersion, and Kerr nonlinearity due to asymmetric gain. The comparison to observations in various semiconductor lasers suggests that the linear chirp is general to self-starting FM combs.
\end{abstract}

DOI: 10.1103/PhysRevLett.123.243902

Optical frequency combs [1,2] are lasers whose spectrum consists of a set of evenly spaced modes that obey a defined phase relation. In the time domain, these lasers emit a signal, which, despite an eventual constant phase drift due to a nonzero carrier envelope offset frequency, is periodic. In the literature, frequency combs are mostly linked to ultrafast lasers that emit short pulses. However, the Fourier theorem states that a comb spectrum is generated by any periodic signal, regardless of its shape. A periodic frequency-modulated (FM) signal is another example that fulfills this criterion. The first studies to generate such a FM laser output trace back to the 1960 s, only a few years after the demonstration of the first laser [3]. An active intracavity phase modulator was used to generate FM oscillations in He-Ne [4] and later in Nd:YAG [5] lasers. Simply from the similarity of the optical spectra to the Bessel amplitudes, it was concluded that FM lasers obey a sinusoidal modulation of the output frequency [6,7] and this picture remained dominant for over 50 years.

Today, FM combs have experienced a renaissance. In 2012, it was shown that quantum cascade lasers (QCLs) could be used to generate combs, whose intensity remains approximately constant [8]. In contrast to the work from the 1960s, the generated FM comb in QCLs is self-starting. The possibility of generating self-starting combs using the nonlinearity provided by the gain medium is particularly appealing for fundamental laser science and the study of self-organization in complex nonlinear systems, but also of great interest for many applications. FM combs can be generated in fast gain media, e.g., QCLs that do not satisfy

Published by the American Physical Society under the terms of the Creative Commons Attribution 4.0 International license. Further distribution of this work must maintain attribution to the author(s) and the published article's title, journal citation, and DOI. the conditions for passive mode locking [9]. They are selfstarting, requiring no additional components, e.g., saturable absorbers, which is interesting for comb generation in interband cascade lasers (ICLs) [10,11]. Both QCLs and ICLs emit in the midinfrared region, which is particularly appealing for dual-comb spectroscopy $[12,13]$.

In this Letter, we provide a rigorous theoretical and numerical study of FM combs that is driven by recent experimental results $[14,15]$. A highly optimized simulation tool was developed to reproduce the experimental results, to identify trends, and to isolate the most relevant terms in the full set of nonlinear coupled differential equations. With this knowledge, we derive a simplified master equation for FM combs. It provides the eagerly awaited intuitive explanation of the phase locking and answers the following questions: (i) What triggers selforganization of the phases in FM combs to overcome chaos? (ii) Why does the linear frequency chirp emerge from this competition, overcoming other solutions? (iii) Why do QCL FM combs lock at low group velocity dispersion (GVD) [16]? (iv) Do FM combs require a fast gain medium?

QCLs posses fast gain dynamics since their carrier lifetimes are on the picosecond scale and considerably shorter than the round-trip time. This is in contrast to the standard slow gain interband semiconductor lasers with lifetimes on the order of nanoseconds. The possibility to generate FM combs with QCLs is mostly explained through their fast gain dynamics. First, the fast gain dynamics lead to strong spatial hole burning. Spatial hole burning originates from the standing wave pattern in the cavity due to the forward and backward propagating components of the electric field. This leads to a static population grating with a period of half the wavelength [17], which effectively increases the gain for the side modes, promoting multimode lasing. In fast gain media, this population grating cannot be washed out efficiently by 
carrier diffusion and its impact is particularly strong. Second, the fast gain dynamics lead to four-wave mixing via the occurrence of population oscillations that respond in antiphase to oscillations of the light intensity $[18,19]$. A modulated intensity saturates the gain more than a constant intensity. Following the maximum emission principle [20], which was recently identified to be essential for FM comb formation [21], amplitude modulations will be suppressed to maximize the output. In fast gain media, this effect is also particularly strong. It is also the reason why a slow gain medium is required for pulse generation. There, the suppression can be compensated and reversed by fast saturable absorption.

The main issue with this concept is that any phase arrangement that minimizes amplitude modulations is equal in energy, which should result in a chaotic phase modulation [22]. Figure 1(a) shows the corresponding numerical simulation result, reproducing the expected pseudorandom multimode operation triggered by spatial hole burning. Experiments, however, clearly show the formation of a distinct periodic pattern and the generation of a frequency comb [14,15]. Figure 1(b) shows the experimental results of a QCL frequency comb with the characteristic linear phase pattern that covers the range from $-\pi$ to $\pi$. Note that we plot the intermodal phases, i.e., the phase difference between adjacent modes. This linear pattern corresponds to parabolic modal phases and a chirped instantaneous frequency.
The suppression of amplitude modulations can be interpreted as repulsive coupling of the intermodal phases. The occurrence of self-organization in repulsively coupled systems can also be found in other fields of research, ranging from splay states in Josephson junctions [24] to cluster states in complex networks [25]. Such phenomena can be explained by additional contributions that induce an imbalance to favor one among many other solutions. Such an imbalance can, for example, be triggered by a finite GVD, as shown by our numerical results in Fig. 1(c). This is particularly surprising, as the experimental observations of FM combs in QCLs were found in dispersion-compensated cavities.

In the following, we explain why the GVD plays such a crucial role and indicate which other effects are required to explain the experimental observations. The starting point is a system of eight coupled nonlinear differential equations that describes the manifold physics of a laser. The system is based on the spatiotemporally resolved Maxwell-Bloch equations in the slowly varying envelope approximation [26,27], which includes the effects of GVD and Kerr nonlinearity that have been mostly omitted previously. The details of the model and all derivations in textbook style can be found in the Supplemental Material [23]. While this full model is capable of a quantitative analysis, it cannot provide an intuitive understanding of the underlying physics.

Laser models with reduced complexity can yield an intuitive interpretation. An example is the Haus master (a)
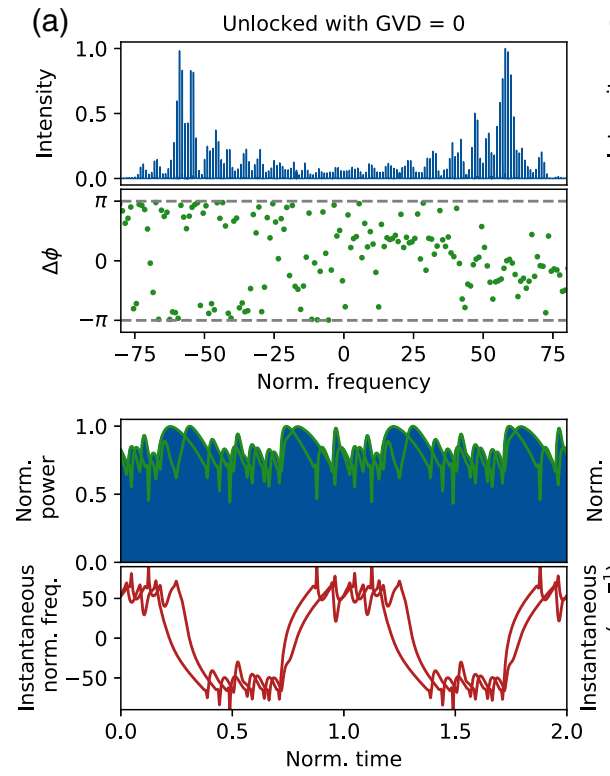

(b)
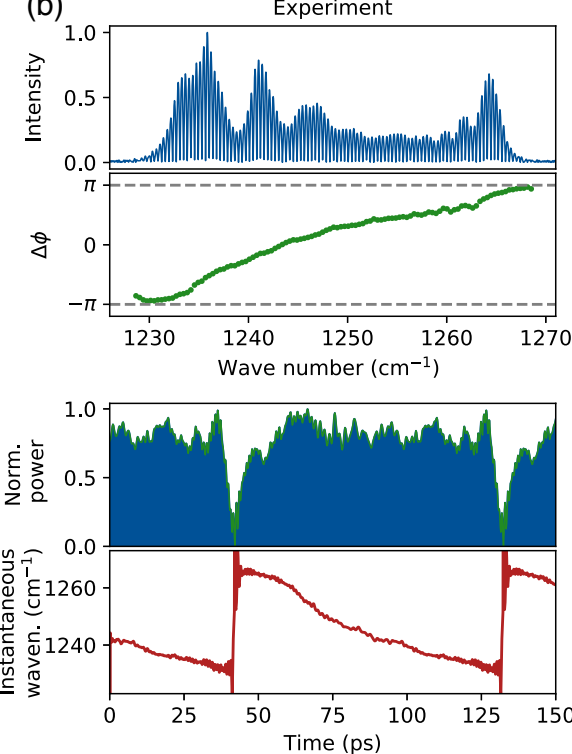

(c)
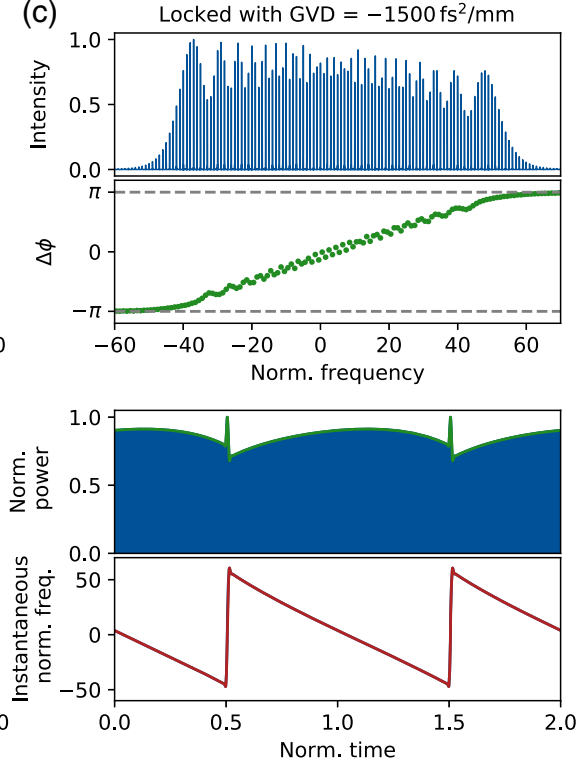

FIG. 1. (a) Simulation of an unlocked laser with both the GVD and Kerr nonlinearity set to zero. (Top) Shows the normalized intensity spectrum with chaotic intermodal phases. (Bottom) Shows the comparison of the time traces of the normalized output intensity and the instantaneous normalized frequency emitted by the laser after 100000 and 150000 cavity round-trips. They do not match, indicating the unlocked state. The frequency and time axes are normalized to the round-trip frequency. (b) Experimental results, reprinted from the data of [15]. (c) Simulated self-phase-locked laser with a nonzero GVD of $-1500 \mathrm{fs}^{2} / \mathrm{mm}$, showing the same characteristics as the experiment: The chirped intermodal phases that cover $2 \pi$ over the spectral span, as well as the suppressed amplitude modulations and the linear frequency chirp in the time traces. All numerical simulations are done using Eq. (1) and the parameters are provided in the Supplemental Material [23], as well as the video of the entire simulation in the case of (a) and (c). 
equation for mode locking with saturable absorbers [9]. Such models utilize the adiabatic approximation to eliminate variables, e.g., the induced polarization and carrier populations. However, with its application, the physics behind FM combs disappears. In the adiabatic elimination, the response of these variables is assumed to be instantaneous, which is equivalent to approximating their transfer function by a constant, e.g., $H(\omega)=a /(1+i \omega T) \approx a$. With this, the information on a remaining small phase delay that can accumulate over hundreds of round-trips is entirely lost. In order to recover sufficient information, we use a Taylor expansion of the form $H(\omega)=a\left(1-i \omega T-\omega^{2} T^{2} \cdots\right)$. This yields a single master equation for lasers with fast gain media

$$
\begin{gathered}
\left(\frac{n}{c} \partial_{t} \pm \partial_{z}\right) E_{ \pm}=\frac{g(P)}{2}\left[E_{ \pm}-T_{2} \partial_{t} E_{ \pm}+T_{2}^{2} \partial_{t}^{2} E_{ \pm}\right] \\
-\frac{g(P) T_{g}}{2 T_{1} P_{\mathrm{sat}}}\left[\left|E_{\mp}\right|^{2} E_{ \pm}-\left(T_{2}+T_{\mathrm{g}}\right)\left|E_{\mp}\right|^{2} \partial_{t} E_{ \pm}\right. \\
\left.-\left(T_{2}+T_{\mathrm{g}}\right) E_{ \pm} E_{\mp} \partial_{t} E_{\mp}^{*}-T_{2} E_{ \pm} E_{\mp}^{*} \partial_{t} E_{\mp}\right] \\
\quad+i \frac{k^{\prime \prime}}{2} \partial_{t}^{2} E_{ \pm}+i \beta\left(\left|E_{+}\right|^{2}+\left|E_{-}\right|^{2}\right) E_{ \pm}-\frac{\alpha_{w}}{2} E_{ \pm}
\end{gathered}
$$

where $c$ is the speed of light, $n$ is the refractive index, $E \pm$ is the right and left propagating complex field envelopes, $T_{1}$, $T_{2}$, and $T_{g}$ are the recovery times of the gain, polarization, and population grating, $k^{\prime \prime}$ is the group velocity dispersion coefficient, $\beta$ is the Kerr nonlinearity coefficient, $\alpha_{w}$ is the waveguide loss, $g(P)=g_{0} /\left(1+P / P_{\text {sat }}\right)$ is the saturated gain, $P_{\text {sat }}$ is the saturation power, and $P=\left|E_{+}\right|^{2}+\left|E_{-}\right|^{2}$ is the normalized power. The highlighted terms correspond to the higher order terms introduced by the Taylor expansion. The full derivation of the master equation is presented thoroughly in the Supplemental Material [23] along with the origin of every term in the equation and its corresponding physical meaning. While we used the master equation for our numerical simulations, we will derive now a reduced form to provide better insight into the most relevant mechanisms.

Aiming for a qualitative explanation of FM combs, we rewrite Eq. (1) in terms of power and phase $E_{ \pm}=$ $\sqrt{P_{ \pm}} \exp \left(i \phi_{ \pm}\right)$and neglect several minor contributions; e.g., terms with $\partial_{t} A$ are smaller than terms with $\partial_{t} \phi$. The reduced master equation for FM combs reads

$$
\begin{gathered}
\left(\frac{n}{c} \partial_{t} \pm \partial_{z}\right) P_{ \pm}=g(P)\left[P_{ \pm}-\frac{T_{2}}{2} \partial_{t} P_{ \pm}\right. \\
\left.-T_{2}^{2} P_{ \pm}\left(\partial_{t} \phi_{ \pm}\right)^{2}\right]-\alpha_{w} P_{ \pm} \\
\left(\frac{n}{c} \partial_{t} \pm \partial_{z}\right) \phi_{ \pm}=-\frac{k^{\prime \prime}}{2}\left(\partial_{t} \phi_{ \pm}\right)^{2}+\beta\left(P_{+}+P_{-}\right) \\
+\frac{g(P)}{2}\left[-T_{2} \partial_{t} \phi_{ \pm}-\frac{T_{g}^{2}}{T_{1}} \frac{P_{\mp}}{P_{\text {sat }}} \partial_{t} \phi_{\mp}\right] .
\end{gathered}
$$

Now it will become clear why we introduced the Taylor expansion (highlighted terms) instead of the adiabatic elimination. As a frequency modulation is essentially a modulation of the phase, FM comb operation is mostly governed by Eq. (3). Without the highlighted terms, the phase dynamics would be lost and with that also the physics of FM combs. The first highlighted term in Eq. (3), proportional to $\partial_{t} \phi_{ \pm}$, dampens phase oscillations, tending toward single mode operation. The second highlighted term proportional to $\partial_{t} \phi_{\mp}$ is due to spatial hole burning. It facilitates multimode operation and is essential for FM comb operation. Without it, the laser would remain in single mode. Simulations that support this claim and a more detailed description of spatial hole burning is provided in the Supplemental Material [23]. The dispersion term proportional to $\left(\partial_{t} \phi_{ \pm}\right)^{2}$ determines the evolution of the cumulative phase shape and favors a convex or concave parabola, depending on the sign of $k^{\prime \prime}$. This results in a chirp in one direction or another. The magnitude of $k^{\prime \prime}$ is directly related to the chirp of the intermodal phases. If it is large enough that the intermodal phases cover the full range of $2 \pi$, the laser can produce a stable periodic output. A further increase makes the intermodal phases slope steeper, which requires a narrowing of the spectrum [Fig. 2(b)], since the range of $2 \pi$ is fixed. The residual amplitude modulation present in both the experiments and simulations (Fig. 1) are mostly due to the second highlighted term in Eq. (2), which is proportional to $P_{ \pm}\left(\partial_{t} \phi_{ \pm}\right)^{2}$.

It is interesting to note that, without the Kerr nonlinearity $(\beta=0)$, the dynamics of FM combs can be described entirely by just Eq. (3), using the steady-state solution of Eq. (2), by setting all time derivatives to zero. However, it remains crucial that the static spatial profile of $P_{ \pm}$is considered in the spatial hole burning term in Eq. (3).

The situation becomes slightly more complex when considering an additional Kerr nonlinearity $\beta$. In that case, Eqs. (2) and (3) are dynamically coupled. The second highlighted term in Eq. (2) represents a source term, forcing $P_{ \pm}$to oscillate in a similar manner. Coupling $P_{ \pm}$back in Eq. (3) through the Kerr term, one sees a similar effect as the GVD, as approximately both are influencing the phase through a term proportional to $\left(\partial_{t} \phi_{ \pm}\right)^{2}$. Figure 2(a) shows the numerical simulation of a FM comb generated by a Kerr nonlinearity with zero GVD. One can observe a slight bending of the intermodal phases, also present in the experimental data [Fig. 1(b)].

The Kerr term mostly originates from a change of the real part of the refractive index with the population distribution. This is connected to an asymmetric spectral gain profile and commonly expressed through a nonzero linewidth enhancement factor (LEF) [18,28]. In the Supplemental Material [23], we show that, in a fast gain medium, a finite LEF leads to a considerable Kerr nonlinearity because the gain is tightly coupled to the intracavity field intensity. On the other hand, in a laser with 

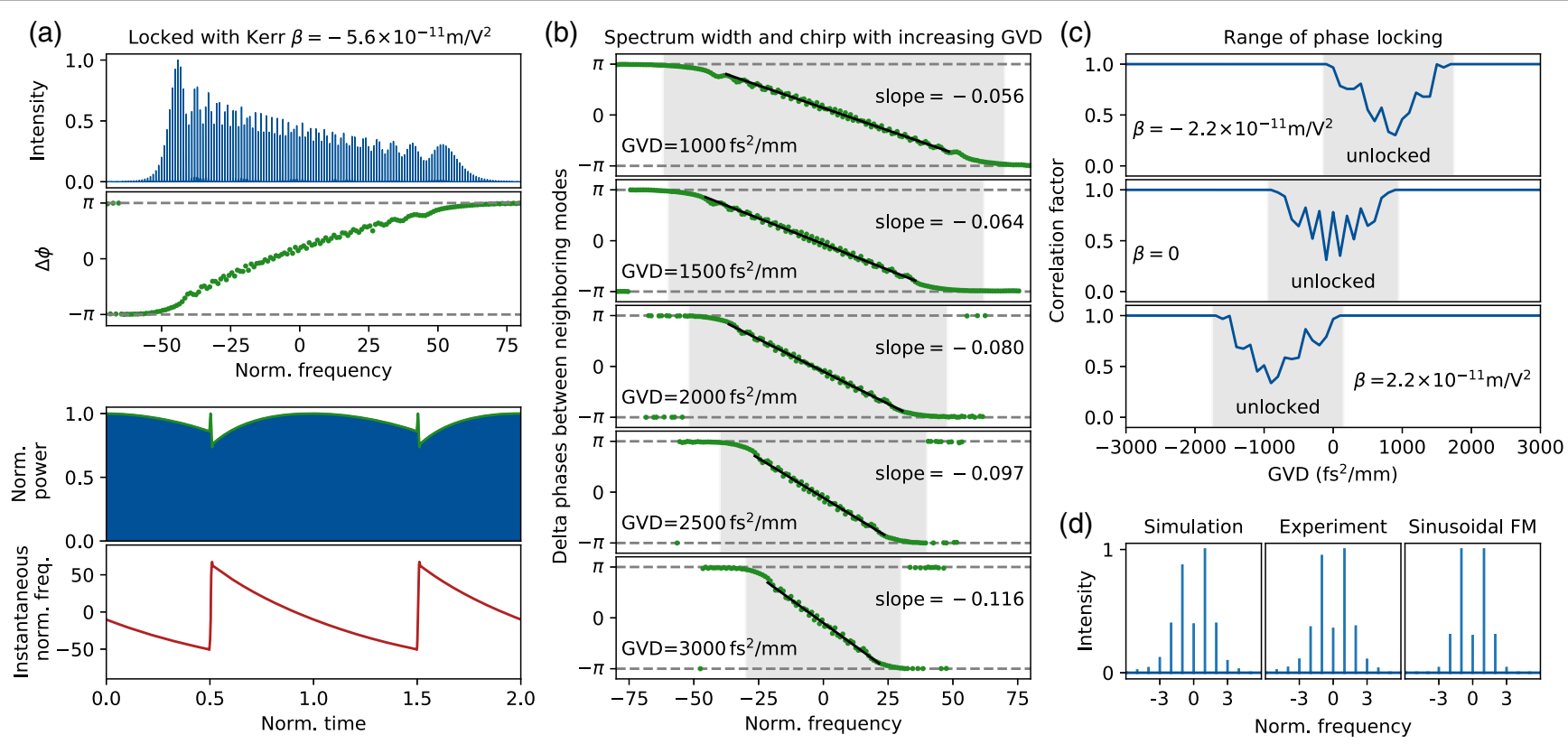

FIG. 2. (a) Simulation of a locked laser in the presence of Kerr nonlinearity $\left(\beta=-5.6 \times 10^{-11} \mathrm{~m} / \mathrm{V}^{2}\right)$ and zero GVD. A video of the entire simulation can be seen in the Supplemental Material [23]. (b) Narrowing of the laser spectrum with zero Kerr nonlinearity and increasing GVD (from 1000 to $3000 \mathrm{fs}^{2} / \mathrm{mm}$ ) resulting in the increase of the intermodal phases slope, which corresponds to the decrease of the chirp. Shaded regions indicate the spectral width. In all cases, the phases cover a range of $2 \pi$. Below GVD $=$ $1000 \mathrm{fs}^{2} / \mathrm{mm}$ the slope is too small to cover the full phase range of $2 \pi$ over the spectral span and the laser remains unlocked. (c) Shifting of the GVD value range required for self-locking, due to a change of the Kerr nonlinearity. (d) Intensity spectrum comparison obtained from our simulation, experiment (recreated from [7]), and a sinusoidal FM given with the Bessel amplitudes. The comparison of the second harmonic spectra is shown in the Supplemental Material [23].

longer carrier lifetime (slower dynamics), this coupling is washed out and the contribution to the Kerr nonlinearity is weaker. A nonzero Kerr nonlinearity or LEF strongly shift the range of GVD required for FM comb operation. This explains why QCL frequency combs have been found close to zero GVD. Figure 2(c) shows the range of GVD required to obtain FM comb operation for three different values of $\beta$ that correspond to realistic values of LEF for QCLs at room temperature [29]. Altering the shape or width of the gain changes the required GVD and thus suggests one should consider this effect in the design of broadband FM combs. It will be interesting to see if the observed behavior at very high values of GVD with the continuous narrowing of the spectrum can also be reproduced in the experiment or, if above a certain threshold, the laser becomes unlocked again due to effects neglected in the reduced model. First attempts of solving the full model for high dispersion again yielded an unlocked state, but the results remain inconclusive due to numerical issues that are known from convectiondiffusion problems. A detailed investigation will be part of future work.

We did not find a reason why FM comb operation should be strictly limited to fast gain media. In QCLs, with their fast dynamics, even a small gain asymmetry leads to a considerable Kerr nonlinearity. In interband semiconductor lasers, this contribution is attenuated due to the slower dynamics, but the asymmetry is much more pronounced. Moreover, dispersion-driven FM comb operation appears to be independent of the gain dynamics. Hence, we believe that FM comb operation with a linear chirp is a general phenomenon. As an example, the presented theory also explains the experimental observations of self-starting FM oscillations in an InGaAsP laser diode [7]. The simulated intensity spectrum with the linear chirp fits the measured modal amplitudes much better than previously assumed Bessel amplitudes [Fig. 2(d)]. Our theory is further consistent with observations in experiments on quantum well [30], quantum dot [31], and quantum dash lasers [32], as well as numerical simulations $[33,34]$ and recent results on the demonstration of FM comb operation in interband cascade lasers [10]. Moreover, by assuming a unidirectional electric field, the derived master equation (1) can be reduced to the well-known complex Ginzburg-Landau equation, which is used in many areas of physics [35], potentially making the impact of this Letter even broader.

In conclusion, we provided detailed insights into the formation of frequency combs in single section lasers without saturable loss. Going beyond the adiabatic approximation, we derived a master equation to explain the physics and identified the most relevant contributions. In accordance with this, a FM comb requires spatial hole burning to trigger multimode operation, gain saturation to suppress 
amplitude modulation, and a minimum, but finite contribution from the GVD or Kerr nonlinearity due to gain asymmetry that gives rise to a chirped output. A minimum amount is required such that the intermodal phases can cover a range of $2 \pi$ over the spectral span to suppress amplitude modulations. Further increase will enforce a narrowing of the spectrum. The presented theory is capable of explaining experimental observations in various types of semiconductor lasers. Furthermore, while the Kerr nonlinearity contribution from the gain asymmetry depends on the laser dynamics, the value of the GVD absolutely does not show such a dependency, indicating that the linear chirp is a general phenomenon behind the nature of FM combs in semiconductor lasers.

This work was supported by the Austrian Science Fund (FWF) within the projects "NanoPlas" (P28914-N27) and "Building Solids for Function" (Project W1243).

*benedikt.schwarz@tuwien.ac.at

[1] T. W. Hänsch, Rev. Mod. Phys. 78, 1297 (2006).

[2] J. L. Hall, Rev. Mod. Phys. 78, 1279 (2006).

[3] T. H. Maiman, Nature (London) 187, 493 (1960).

[4] S. E. Harris and R. Targ, Appl. Phys. Lett. 5, 202 (1964).

[5] D. Kuizenga and A. Siegman, IEEE J. Quantum Electron. 6, 673 (1970).

[6] S. Harris and O. McDuff, IEEE J. Quantum Electron. 1, 245 (1965).

[7] L. Tiemeijer, P. Kuindersma, P. Thijs, and G. Rikken, IEEE J. Quantum Electron. 25, 1385 (1989).

[8] A. Hugi, G. Villares, S. Blaser, H. C. Liu, and J. Faist, Nature (London) 492, 229 (2012).

[9] H. Haus, IEEE J. Quantum Electron. 11, 323 (1975).

[10] B. Schwarz, J. Hillbrand, M. Beiser, A. M. Andrews, G. Strasser, H. Detz, A. Schade, R. Weih, and S. Höfling, Optica 6, 890 (2019).

[11] M. Bagheri, C. Frez, L. A. Sterczewski, I. Gruidin, M. Fradet, I. Vurgaftman, C. L. Canedy, W. W. Bewley, C. D. Merritt, C. S. Kim, M. Kim, and J. R. Meyer, Sci. Rep. 8, 3322 (2018).

[12] G. Villares, A. Hugi, S. Blaser, and J. Faist, Nat. Commun. 5, 5192 (2014).

[13] L. A. Sterczewski, J. Westberg, M. Bagheri, C. Frez, I. Vurgaftman, C. L. Canedy, W. W. Bewley, C. D. Merritt, C. S. Kim, M. Kim, J. R. Meyer, and G. Wysocki, Opt. Lett. 44, 2113 (2019).
[14] M. Singleton, P. Jouy, M. Beck, and J. Faist, Optica 5, 948 (2018).

[15] J. Hillbrand, A. M. Andrews, H. Detz, G. Strasser, and B. Schwarz, Nat. Photonics 13, 101 (2019).

[16] G. Villares, S. Riedi, J. Wolf, D. Kazakov, M. J. Süess, P. Jouy, M. Beck, and J. Faist, Optica 3, 252 (2016).

[17] C. L. Tang, H. Statz, and G. deMars, J. Appl. Phys. 34, 2289 (1963).

[18] G. P. Agrawal, J. Opt. Soc. Am. B 5, 147 (1988).

[19] T. S. Mansuripur, C. Vernet, P. Chevalier, G. Aoust, B. Schwarz, F. Xie, C. Caneau, K. Lascola, C. en Zah, D. P. Caffey, T. Day, L. J. Missaggia, M. K. Connors, C. A. Wang, A. Belyanin, and F. Capasso, Phys. Rev. A 94, 063807 (2016)

[20] C. L. Tang and H. Statz, J. Appl. Phys. 38, 2963 (1967).

[21] M. Piccardo, P. Chevalier, B. Schwarz, D. Kazakov, Y. Wang, A. Belyanin, and F. Capasso, Phys. Rev. Lett. 122, 253901 (2019).

[22] N. Henry, D. Burghoff, Y. Yang, Q. Hu, and J. B. Khurgin, Opt. Eng. 57, 1 (2017).

[23] See Supplemental Material at http://link.aps.org/ supplemental/10.1103/PhysRevLett.123.243902 for the derivation and physical interpretation of the theoretical model, videos of the simulated intra-cavity dynamics and additional numerical results.

[24] S. H. Strogatz and R. E. Mirollo, Phys. Rev. E 47, 220 (1993).

[25] L. M. Pecora, F. Sorrentino, A. M. Hagerstrom, T. E. Murphy, and R. Roy, Nat. Commun. 5, 4079 (2014).

[26] K. Shimoda, Introduction to Laser Physics (Springer, Berlin, 1984).

[27] Y. Wang and A. Belyanin, Opt. Express 23, 4173 (2015).

[28] G. Gray and G. Agrawal, IEEE Photonics Technol. Lett. 4, 1216 (1992).

[29] T. Aellen, R. Maulini, R. Terazzi, N. Hoyler, M. Giovannini, J. Faist, S. Blaser, and L. Hvozdara, Appl. Phys. Lett. 89, 091121 (2006).

[30] K. Sato, Electron. Lett. 37, 763 (2001).

[31] M. J. R. Heck, E. A. J. M. Bente, B. Smalbrugge, Y.-S. Oei, M. K. Smit, S. Anantathanasarn, and R. Nötzel, Opt. Express 15, 16292 (2007).

[32] R. Rosales, S. G. Murdoch, R. Watts, K. Merghem, A. Martinez, F. Lelarge, A. Accard, L. P. Barry, and A. Ramdane, Opt. Express 20, 8649 (2012).

[33] M. Gioannini, P. Bardella, and I. Montrosset, IEEE J. Sel. Top. Quantum Electron. 21, 698 (2015).

[34] M. Dong, N. M. Mangan, J. N. Kutz, S. T. Cundiff, and H. G. Winful, IEEE J. Quantum Electron. 53, 1 (2017).

[35] I. S. Aranson and L. Kramer, Rev. Mod. Phys. 74, 99 (2002). 